\title{
Application of Partial Least Square on Quantitative Analysis of L-, D-, and DL-Tartaric Acid by Terahertz Absorption Spectra
}

\author{
Rika Nishikiori, ${ }^{a}$ Mariko Yamaguchi, ${ }^{b}$ Keisuke Takano, ${ }^{b}$ Tokujiro Enatsu, ${ }^{b}$ Masahiko Tani,${ }^{b}$ \\ U. Chandimal de Silva, ${ }^{c}$ Norihito Kawashita, ${ }^{d}$ Tatsuya TaKagi,${ }^{d}$ Shotaro Morimoto, ${ }^{a}$ \\ Masanori HANGYO, ${ }^{b}$ and Masaya KAWASE ${ }^{*}, a$ \\ ${ }^{a}$ Faculty of Pharmaceutical Sciences, Osaka Ohtani University; 3-11-1 Nishikiori-kita, Tondabayashi, Osaka 584-8540, \\ Japan: ${ }^{b}$ Institute of Laser Engineering, Osaka University; 2-6 Yamada-oka, Suita, Osaka 565-0871, Japan: ${ }^{c}$ Thailand- \\ Japan Research Collaboration Center on Emerging and Re-emarging Infection (RCC-ERI); 6th Fl., Building 10, \\ Department of Medical Sciences, Ministry of Public Health, Tiwanon Road, Muang, Nonthaburi 11000, Thailand: and \\ ${ }^{d}$ Graduate School of Pharmaceutical Sciences, Osaka University; 1-6 Yamada-oka, Suita, Osaka 565-0871, Japan.
}

Received October 24, 2007; accepted January 7, 2008; published online January 10, 2008

\begin{abstract}
Absorption spectra of polycrystalline L-, D-, and DL-tartaric acid have been measured by terahertz time domain spectroscopy (THz-TDS). Different absorption bands are observed for DL-tartaric acid and its enantiomers (L- and D-tartaric acid). This result shows that the THz-TDS can be used for distinguishing between DL-tartaric acid and enantiomers ( $L$ - and D-tartaric acid). Moreover, partial least square (PLS) can be found to improve the quantitation of $\mathrm{L}-$ tartaric acid in $\mathrm{L}$ - and DL-tartaric acid mixture by THz-TDS.
\end{abstract}

Key words terahertz time domain spectroscopy; tartaric acid; partial least square

Various applications of terahertz (THz) electromagnetic wave have been reported in many fields, such as medical diagnosis, pharmaceutical analysis, and security enhancement. ${ }^{1-8)}$ Absorption spectra in the $\mathrm{THz}$ range provide rich information for both intermolecular and intramoleculer interactions, such as inter-ring interactions of disaccharides. ${ }^{6}$ )

Absorption spectra in the $\mathrm{THz}$ range is very sensitive to the difference in the crystal structure, ${ }^{9)}$ and isapplied to study the polymorphs of medicines. ${ }^{7)}$ The $\mathrm{THz}$ time domain spectroscopy (THz-TDS) using the THz pulses generated by femtosecond laser pulses is useful to measure the optical properties in wave number (frequency) range below $100 \mathrm{~cm}^{-1}$ $(3 \mathrm{THz})$, and gives a better signal-to-noise ratio than the farinfrared Fourier transform (FIR FT-IR) spectroscopy. ${ }^{10)} \mathrm{THz}$ spectra is sensitive to the assembly state of molecules. The assembly state is considered to be different between optical isomers. Because of such characteristics of $\mathrm{THz}$ spectra, they could used to distinguish between the optical isomers. Yamaguchi et al. ${ }^{11)}$ reported that absorption spectra of $\mathrm{THz}$ range could distinguish DL- and L-alanine (or D-alanine), and suggested the possibility in determination of enantiometric composition of amino acids.

In this paper, we report the measurement of the absorption spectra of polycrystalline D-, L-, and DL-tartaric acid by THzTDS. It was found that the absorption spectra showed no significant difference between $\mathrm{D}$ - and L-tartaric acid. On the other hand, the absorption spectra showed a remarkable difference between L- (or D-) and DL-tartaric acid. Based on the spectral difference, it was demonstrated that a quantitative analysis of L-tartaric acid in the mixture of $\mathrm{L}$ - and DL-tartaric acid is possible.

\section{Experimental}

Apparatus The absorption spectra were measured in the frequency range from 0 to $3 \mathrm{THz}$ using a standard THz-TDS setup. ${ }^{12)} \mathrm{A}$ mode-locked Ti:sapphire laser was used as the pump source. The emitter was a dipoletype photoconductive antenna ${ }^{13)}$ excited by laser pulses with the average of $15 \mathrm{~mW}$. The bias voltage applied to the emitter photoconductive antenna was $35 \mathrm{~V}_{\mathrm{p}-\mathrm{p}}$ and modulated at about $10 \mathrm{kHz}$. The $\mathrm{THz}$ radiation from the emitter was collected and focused on the sample by a parabolic mirror. The THz radiation transmitted through the sample was collected and focused by another parabolic mirror on to the detector photoconductive antenna, which was a bowtie-type on the low-temperature grown GaAs (LT-GaAs) substrate. ${ }^{13)}$ The detector antenna was triggered by probe pulses $(10 \mathrm{~mW})$. Hyper-spherical Si substrate lenses were used for both the emitter and detector antennas to reduce the total reflection in the GaAs substrates and increase the beam collection efficiency. The photoconductive current signal was detected with a lock-in amplifier and its time-domain signal was obtained by scanning the optical delay of probe pulse. By Fourier transforming the time-domain signal, the amplitude spectra were obtained. To reduce the absorption due to water vapor in the ambient air, the $\mathrm{THz}$ beam path was filled with dry air.

Sample Preparation L- and DL-Tartaric acid were mixed with several predetermined ratios, and the $50 \mathrm{mg}$ of mixtures were pressed under a pressure of $150 \mathrm{~kg} / \mathrm{m}^{2}$ to form pellets with a diameter of $13 \mathrm{~mm}$. The thickness of samples was measured and used to derive the absorption coefficient of each sample.

Reagents and Chemicals All chemicals were obtained from commercial sources, and used without further purification.

\section{Results and Discussion}

Figure 1 shows the spectra of the absorption coefficient for L-, D-, and DL-tartaric acid. All spectra show several absorption peaks on the absorption continuum which increases with frequency. The $\mathrm{THz}$ radiation hardly transmitted through the sample at frequencies higher than $2.5 \mathrm{THz}$, and the signal intensity became very weak at frequencies higher than $2.5 \mathrm{THz}$. One main absorption band is observed at $1.09 \mathrm{THz}$ for L- and D-tartaric acid. This absorption band is not observed for DL-tartaric acid. It is considered that the difference between spectra of DL-tartaric acid and L- (or D-) tartaric acid is caused by following reasons. THz-TDS spectra sharply reflect both intermolecular vibrations and phonon frequency. These modes are affected by the difference of symmetry of crystal and the difference of intermolecular interactions, such as $\mathrm{D}-\mathrm{D}, \mathrm{L}-\mathrm{L}$ and $\mathrm{D}-\mathrm{L}$. It is considered that both intermolecular vibrations and phonon frequency are the same in $\mathrm{D}-$ and $\mathrm{L}$ tartaric acids, but that both intermolecular vibrations and phonon frequency of DL-tartaric acid is different from those of L- (or D-) tartaric acid. Thus, spectra in Fig. 1 were obtaind. This result suggests that THz-TDS could distinguish 


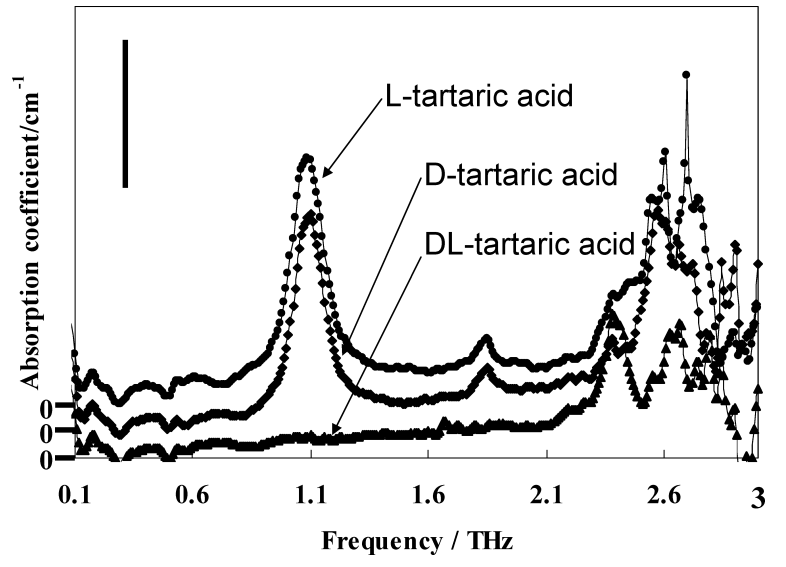

Fig. 1. Absorption Spectra of L-, D-, and DL-Tartaric Acids

Zero position of each spectrum is shown on vertical axis. Scale bar shows 5 of absorption coefficient.

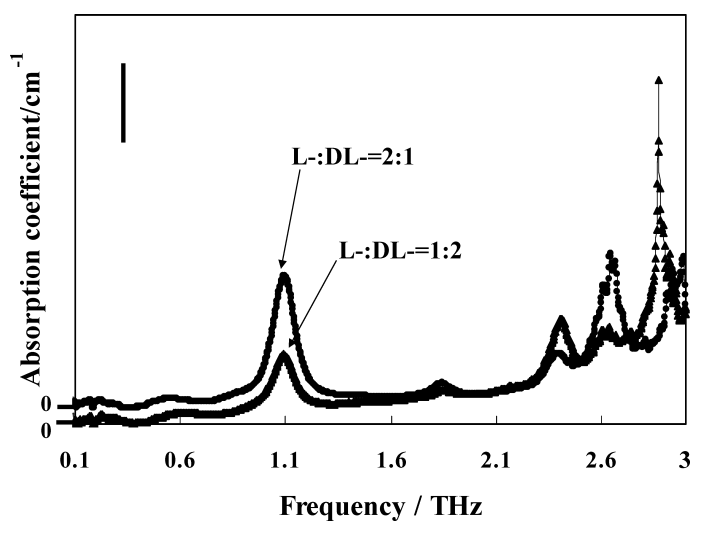

Fig. 2. Absorption Spectra of Mixture of L- and DL-Tartaric Acids

Molar ratio of L- to DL-tartaric acid was 2 and 0.5 . Zero position of each spectrum is shown on vertical axis. Scale bar shows 5 of absorption coefficient.

between DL-tartaric acid and L- (or D-) tartaric acid.

In Fig. 2, two spectra, whose molar ratio of L- to DL-tartaric acid was 2 and 0.5 , are shown. It seems that the absorption coefficient of the band at $1.09 \mathrm{THz}$ depends linearly on the molar ratio of L- to DL-tartaric acid. This tendency is also observed in other peaks. The composition dependence of the absorption coefficient of the band at $1.09 \mathrm{THz}$ was examined more quantitatively. It should be noted that since the absorption intensity depends on the sample thickness, which slightly varies from the sample to sample, a thickness-independent quantity, such as the absorption coefficient, needs to be used in the quantitative analysis. The calculated composition dependence is shown in Fig. 3. The regression line on the figure showed a reasonably good fitting, and the decision coefficient of the regression equation was 0.95 . However, it seems that straight line recurrence is not appropriate, because data positions are clearly deviated from the regression line at the right end of the mixture ratio in Fig. 3. The reasons of deviation from the regression line at the right end of the mixture ratio are that the spectra also have continuous absorption components which increase with frequency. Signal intensity of such components is different between samples. Such difference is occurred for the change of a sample state in mixing caused by the difference of density and particle equality. It was found that the error in the quantitative analy-

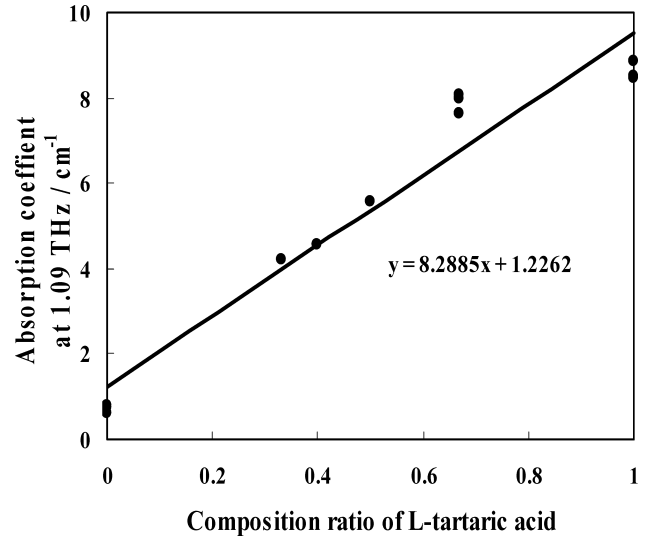

Fig. 3. Dependence of the Absorption Intensity of the Band at $1.09 \mathrm{THz}$ of the Composition Ratio of L-Tartaric Acids

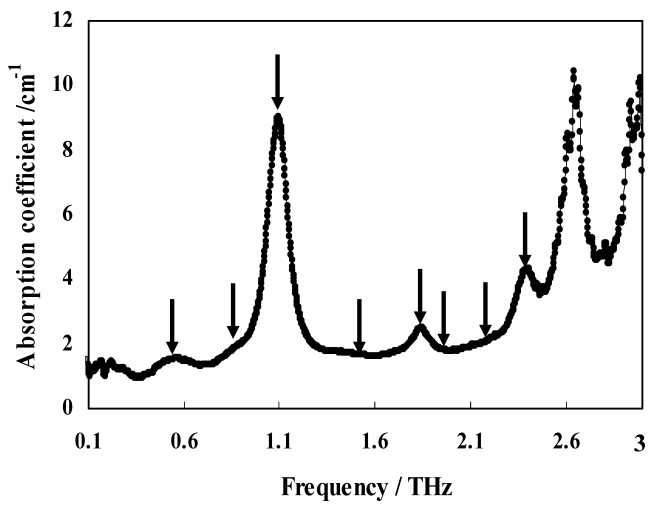

Fig. 4. Employed Absorption Bands for MLR, PLS and QPLS Arrows show the employed bands.

Table 1. Summary of the Employed Multiple Regression

\begin{tabular}{lc}
\hline \hline Method & Decision coefficient \\
\hline MLR & 0.990 \\
PLS & 0.994 \\
QPLS & 0.995 \\
\hline
\end{tabular}

sis increased, when the only one absorption coefficient (at $1.09 \mathrm{THz}$ ) was used, because of deviation from the regression line. Therefore, improvement of quantitation was attempted by some multiple regression using eight spectral data points shown by arrows in Fig. 4. Multiple linear regression (MLR), partial least squares (PLS), ${ }^{14)}$ and quadratic PLS (QPLS) ${ }^{14)}$ were employed for the regression analysis. QPLS is a nonlinear regression method. Chemish system, which has been developed by Funatsu and co-workers was used in the analysis. ${ }^{14)}$ The obtained decision coefficients were listed in Table 1. These three methods gave a better decision coefficient. This result indicates that the absorption coeficients at $0.95 \mathrm{THz}, 2.00 \mathrm{THz}$, and $2.38 \mathrm{THz}$ are also necessary for more reliable quantitative analysis. It is also found that PLS and QPLS are better than MLR for quantitative analysis based on the THz-TDS absorption spectra. In general, the spectra obtained in this study are very simple, but the spectra of other chemicals could be more complex. THz-TDS absorption spectra often have very complex base line. This 
property is very serious problem in quantitative analysis. If base line is not considered, the obtained result contains a large error. PLS and QPLS make the quantitative analysis model using both spectrum peaks and base line, and they present better quantitative analysis model than that by MLR. Thus, PLS and QPLS can also dissolve the above problem in quantitative analysis based on the THz-TDS absorption spectra. PLS and QPLS methods are considered to be promising in quantitative analysis of more complex spectra of $\mathrm{THz}-$ TDS.

Applications of $\mathrm{THz}-\mathrm{TDS}$ are expanding to various fields, ${ }^{15)}$ as a useful analytical tool. Therefore, to take full advantage of this method, it becomes more and more important to develop efficient analytical methods, which can draw important information from THz-TDS spectra. This study shows that THz-TDS is a promising tool for quantification of optically active compounds when used with proper analytical methods, such as PLS and QPLS.

\section{References}

1) Nagel M., Richter F., Haring-Bolivar P., Kurtz H., Phys. Med. Biol., 48, 3625-3636 (2003).

2) Woodward R. M., Wallace V. P., Pye R. J., Cole B. E., Amone D. D.,
Linfield E. H., Pepper M., J. Invest. Dermatol., 120, 72-78 (2003).

3) Yamamoto K., Yamaguchi M., Miyamaru F., Tani M., Hangyo M., Ikeda T., Matsushita A., Koide K., Tatsuno M., Miyami Y., Jpn. J. Appl. Phys., 43, L414-L417 (2004).

4) Kawase K., Ogawa Y., Watanabe Y., Opt. Express, 11, 2549-2554 (2003).

5) Yamamoto K., Yamaguchi M., Tani M., Hangyo M., Teramaru S., Isu T., Tomira N., Appl. Phys. Lett., 85, 5194-5196 (2004).

6) Hineno M., Yoshinaga H., Spectrochim. Acta A, 30, $411-416$ (1974).

7) Taday P. F., Bradley I. V., Arnone D. D., Pepper M., J. Pharm. Sci., 92, $831-838$ (2003).

8) Walther M., Plochocka P., Fisher B., Helm H., Uhd Jepsen P., Biopolymers, 67, 310-313 (2002).

9) Walther M., Plochocka P., Fisher B., Helm H., Uhd Jepsen P., Chem. Phys. Lett., 332, 389-395 (2000).

10) Grischkowsky D., Keiding S., Exter M., Fattinger Ch., J. Opt. Sci. Am. $B, 7,2006-2015$ (1990).

11) Yamaguchi M., Miyamaru F., Yamamoto K., Tani M., Hangyo M., Appl. Phys. Lett., 86, 53903-1-53903-3 (2005).

12) Hangyo M., Nagashima T., Nashima S., Meas. Sci. Technol., 13, $1727-1738$ (2002).

13) Tani M., Matsuura S., Sakai K., Appl. Opt., 36, 7853-7859 (1997).

14) Tanada T., Arakawa M., Nishimura R., Funatsu K., J. Comput. Aid. Chem., 1, 35-46 (2000).

15) Hangyo M., Tani M., Nagashima T., Int. J. Infrared Milli. Waves, 26, $1661-1690$ (2005). 